\title{
Explicit Solution of Reinsurance-Investment Problem for an Insurer with Dynamic Income under Vasicek Model
}

\author{
De-Lei Sheng \\ Faculty of Applied Mathematics, Shanxi University of Finance and Economics, Taiyuan 030006, China \\ Correspondence should be addressed to De-Lei Sheng; tjhbsdl@126.com
}

Received 14 October 2015; Accepted 22 December 2015

Academic Editor: Doojin Ryu

Copyright (C) 2016 De-Lei Sheng. This is an open access article distributed under the Creative Commons Attribution License, which permits unrestricted use, distribution, and reproduction in any medium, provided the original work is properly cited.

\begin{abstract}
Unlike traditionally used reserves models, this paper focuses on a reserve process with dynamic income to study the reinsuranceinvestment problem for an insurer under Vasicek stochastic interest rate model. The insurer's dynamic income is given by the remainder after a dynamic reward budget being subtracted from the insurer's net premium which is calculated according to expected premium principle. Applying stochastic control technique, a Hamilton-Jacobi-Bellman equation is established and the explicit solution is obtained under the objective of maximizing the insurer's power utility of terminal wealth. Some economic interpretations of the obtained results are explained in detail. In addition, numerical analysis and several graphics are given to illustrate our results more meticulous.
\end{abstract}

\section{Introduction}

Existing literatures investigate the optimal reinsuranceinvestment problem only from the view of insurer but lack consideration to balance the interests of both insurance company and policy-holders. An effective way to balance both profits of insurer and policy-holders is reward budget. The reward budget is a dynamic item which can be subtracted from the actuarial premium income using the classic premium principle. As a consequence, insurer's premium income is dynamic, which is given by an actuarial income under classic premium principle minus a dynamic reward budget item.

Portfolio optimization is a very interesting problem, which attracted a lot of research work. Cao and $\mathrm{Xu}$ [1] derived the forms of proportional and excess-of-loss reinsurance contracts under the assumption that investment fund follows the logarithm-normal distribution. Cao and Zeng [2] studied the optimal reinsurance-investment problem in order to minimize the probability of ruin. However, only a handful of research works studied the portfolio optimization problem under the stochastic interest rates during the past twenty years. Grasselli [3] studies an investment problem for the HARA utility functions assuming the interest rates are described with the Cox-Ingersoll-Ross dynamics. Under the same stochastic interest rate model, $\mathrm{Li}$ and $\mathrm{Wu}$ [4] obtained an optimal policy in closed form in order to maximize a power utility for the optimal investment problem. When the interest rate is described with an ergodic Markov diffusion process, Pang [5] considered a portfolio optimization problem on an infinite time horizon by maximizing the infinite horizon expected discounted log utility of consumption. If the randomness of the interest rates and inflation are considered at the same time, Munk and Rubtsov [6] obtained a solution in closed form of stockbond-cash portfolio problem for a risk- and ambiguityaverse investor. In addition, Korn and Kraft [7] considered just an investment problem with stochastic interest rates; however, they assumed that an investor can invest on several assets, such as savings account, stocks, and bonds, in order to maximize an insurer's utility of terminal wealth without the usual Lipschitz assumptions. Similarly, Hainaut [8] also considered the financial market with a variety of assets, cash, stocks, and a zero coupon bond, and investigated the problem of dynamic asset allocation assuming the dynamics of the instantaneous short rates being driven by a Hull and White 
model. But all these previous research works did not consider the insurer having a dynamic income, they have ignored the insurers need to identify preferred customers for promotional campaigns or for customer favourable activities in a variety of forms. Such neglected problems directly lead to our study of the dynamic reward budget, which makes the income of insurance company become dynamic.

This paper focuses on a reinsurance-investment problem with dynamic income for an insurer under Vasicek stochastic interest rate model. The dynamic income of an insurer is given by the remainder after a dynamic reward budget being subtracted from the insurer's net premium which is calculated according to expected premium principle. Reward budget is an effective measure to attract more customers so that the total premium income may increase faster with time $t$. It is also an effective way to balance the profit of both insurance company and policy-holders. A Hamilton-JacobiBellman equation is established and the explicit solution for the HJB equation is obtained by maximizing the insurer's power utility of terminal wealth. At last, some numerical analysis and graphics are given to show the impacts of different parameters. Many interesting facts and laws hidden behind the formulas become very clear and more easy to understand.

The subsequent parts of this paper are organized as follows. Section 2 gives some models and a general framework will be used hereinafter. In Section 3, two Hamilton-JacobiBellman equations are established for both cases of stochastic interest rate and of constant interest rate, respectively. The explicit solutions are given with the constant relative risk aversion function and strategies of two different cases are compared. In addition, the economic interpretations of the obtained results are given in the last subsection of this part. Section 4 provides some numerical analysis and graphics.

\section{Formulation of the Model}

Throughout this paper, $\left(\Omega, \mathscr{F}, P,\left\{\mathscr{F}_{t}\right\}_{0 \leq t \leq T}\right)$ denotes a complete probability space satisfying the usual condition, where $T>0$ is a finite constant representing the investment time horizon; $\mathscr{F}_{t}$ stands for the information available until time $t$.

2.1. The Financial Market. We consider a financial market in which transaction amounts are so small that we can consider they have no influence on the prices, with the market being no arbitrage, frictionless, and traded continuously.

Without loss of generality, we assume that the financial market is composed of two kinds of assets: cash and equities. For the sake of simplicity, we will only consider one equity asset which can indeed represent the index of a stock market.

The instantaneous risk-free rate $r(t)$ followed by the stochastic interest rate models of constant volatility, which is described by the Vasicek [9] model:

$$
\mathrm{d} r_{t}=\left(\beta-\alpha r_{t}\right) \mathrm{d} t+\delta \mathrm{d} \widehat{W}(t)
$$

where $\widehat{W}(t)$ is a standard Brownian motion and $\beta>0, \alpha>0$, and $\delta>0$ are constants.
The price $S_{0}(t)$ of the cash asset is given by

$$
\frac{\mathrm{d} S^{0}(t)}{S^{0}(t)}=r(t) \mathrm{d} t, \quad S^{0}(0)=1 .
$$

Here $r(t)$ is the stochastic interest rate.

The stock market index price $S(t)$ at time $t \geq 0$ can be described by the model:

$$
\frac{\mathrm{d} S(t)}{S(t)}=[r(t)+u(t)] \mathrm{d} t+v \mathrm{~d} \widetilde{W}(t), \quad S(0)=s_{0},
$$

where $u(t)$ is another positive real-valued function and the constant $v>0$ denotes the volatility rate of risk asset. $\rho_{2} \epsilon$ $[-1,1]$ is the correlation coefficient of two Brownian motions $\widehat{W}(t)$ and $\widetilde{W}(t)$ and $E[\widehat{W}(t), \widetilde{W}(t)]=\rho_{2} \cdot t$.

In model (3), $u(t)>0$ is a natural hypothesis, which means that the investment on stock can make more money than investing on cash asset, which is consistent with the economic principle of high-risk high yield. In fact, only when $u(t)>0$, more people are willing to invest on equities. For simplicity, we also can take the positive real-valued function $u(t)$ as a positive constant $u>0$, which does not bring substantial impact on the problem investigated in this paper.

2.2. The Surplus Process of Insurer. We use the classical Cramér-Lundberg model to describe the surplus process of insurer:

$$
\mathrm{d} R(t)=c \mathrm{~d} t-\mathrm{d}\left(\sum_{i=1}^{N(t)} Y_{i}\right)
$$

where $N(t)$ is a homogeneous Poisson process with intensity $\lambda$ and the claim sizes $\left\{Y_{i}, i \geq 1\right\}$ are independent and identically distributed positive random variables with $E\left[Y_{i}\right]=$ $\mu$ and $E\left[Y_{i}^{2}\right]=\sigma^{2}$. According to the expected value premium principle, the pure premium rate of insurer is $c=(1+\theta) \lambda \mu$ and $\theta>0$ is the safety loading of insurer.

The diffusion approximation of the compound Poisson process $\mathrm{d}\left(\sum_{i=1}^{N(t)} Y_{i}\right)$ is given by

$$
\mathrm{d}\left(\sum_{i=1}^{N(t)} Y_{i}\right)=\lambda \mu \mathrm{d} t-\sqrt{\lambda} \sigma \mathrm{d} W(t),
$$

where $W(t)$ is a standard Brownian motion, which is independent with $\widetilde{W}(t)$. Moreover, we also assume $E[W(t), \widehat{W}(t)]=\rho_{1} \cdot t, \rho_{1} \in[-1,1]$ denoting the correlation coefficient of $W(t)$ and $\widehat{W}(t)$.

In order to avoid unbearable risk, the insurance company can buy proportional reinsurance to transfer part of the potential risk to reinsurance company. The insurer's retention proportion is $p \in[0,1]$, and the remaining $1-p$ will be borne by the reinsurer. $\eta>0$ is the safety loading of reinsurer:

$$
\begin{aligned}
\mathrm{d} R(t)= & {[c-(1+\eta)(1-p) \lambda \mu] \mathrm{d} t } \\
& -p[\lambda \mu \mathrm{d} t-\sqrt{\lambda} \sigma \mathrm{d} W(t)] \\
= & {[(1+\theta) \lambda \mu-(1+\eta)(1-p) \lambda \mu-p \lambda \mu] \mathrm{d} t } \\
& +p \sqrt{\lambda} \sigma \mathrm{d} W(t) .
\end{aligned}
$$


2.3. The Insurer's Wealth Process with Reward Budget. The proportion of wealth invested on stock asset is denoted by $\pi(t)$ which is a progressively measurable real function, $X(t)$ with $X(0)=x_{0}$ represents the wealth of the insurer, and then we have

$$
\begin{aligned}
\mathrm{d} X(t) & \\
= & \mathrm{d} R(t)+\pi(t) X(t) \frac{\mathrm{d} S(t)}{S(t)} \\
& +(1-\pi(t)) X(t) \frac{\mathrm{d} S^{0}(t)}{S^{0}(t)} \\
= & {[(\theta-\eta) \lambda \mu+\eta p \lambda \mu+(\pi(t) u(t)+r(t)) X(t)] \mathrm{d} t } \\
& +p \sqrt{\lambda} \sigma \mathrm{d} W(t)+v \pi(t) X(t) \mathrm{d} \widetilde{W}(t) .
\end{aligned}
$$

A notation $d=(\eta-\theta) \lambda \mu / \eta p \lambda \mu=(\eta-\theta) / \eta p=(1-$ $\theta / \eta)(1 / p)$ and $(\eta-\theta) \lambda \mu=d \eta p \lambda \mu$, where $d$ is an important constant which indicates the benefit game on safety loading of insurer and reinsurer. The new notation $d$ introduced here is interesting, existing for every combination of an insurance contract with a proportional reinsurance transaction. If $\eta=\theta$, then $d=0$, with such proportional reinsurance transaction being more favourable for insurer. The actual reinsurance transaction must balance both benefits of insurer and reinsurer, so that $0<d<1$ holds invariably.

The reward budget at a fixed experience share is deducted from premium income at each time $t$ :

$$
\begin{aligned}
& \mathrm{d} X(t)=[(c-k X(t))-(1+\eta)(1-p) \lambda \mu] \mathrm{d} t \\
& -p[\lambda \mu \mathrm{d} t-\sqrt{\lambda} \sigma \mathrm{d} W(t)]+\pi(t)(1-k) X(t) \frac{\mathrm{d} S(t)}{S(t)} \\
& +(1-\pi(t))(1-k) X(t) \frac{\mathrm{d} S^{0}(t)}{S^{0}(t)}=[(1-d) \eta p \lambda \mu \\
& +(1-k) X(t) r(t)-k X(t) \\
& +\pi(t)(1-k) X(t) u(t)] \mathrm{d} t+p \sqrt{\lambda} \sigma \mathrm{d} W(t)+\pi(t) \\
& \quad(1-k) X(t) v \mathrm{~d} \widetilde{W}(t) .
\end{aligned}
$$

\section{Main Results}

In this subsection, we consider the reinsurance-investment problem with stochastic interest rate. Following Merton [10], we use an expected utility maximization criterion and assume that the insurer's objective is to maximize the expected utility from terminal wealth, where the utility $U(x)$ is a power utility (constant relative risk aversion) function:

$$
U(x)=x^{q}, \quad q \in(0,1) .
$$

This utility function plays a vital role in actuarial mathematics and insurance practice.

Using the definition of Arrow-Pratt measure of absolute risk-aversion (ARA)

$$
\operatorname{ARA}(x)=-\frac{U^{\prime \prime}(x)}{U^{\prime}(x)}
$$

the coefficient of absolute risk aversion of power utility $U(x)$ is

$$
\operatorname{ARA}(x)=-\frac{q(q-1) x^{(q-2)}}{q x^{(q-1)}}=\frac{1-q}{x}
$$

which decreases with the increase of wealth.

According to the definition of Arrow-Pratt measure of relative risk-aversion (RRA)

$$
\operatorname{RRA}(x)=x \cdot \operatorname{ARA}(x)=-x \frac{U^{\prime \prime}(x)}{U^{\prime}(x)},
$$

the coefficient of relative risk aversion of power utility $U(x)$ is

$$
\operatorname{RRA}(x)=x \cdot \operatorname{ARA}(x)=-x \frac{q(q-1) x^{(q-2)}}{q x^{(q-1)}}=1-q
$$

which does not change with the level of wealth.

Understanding the economic meaning of the two expressions, $(1-q)$ and $(1-q) / x$, is important to understand the results in this paper.

A strategy $\Delta=(p(t), \pi(t))$ is said to be admissible, if $\forall t \in[0, T], \Delta$ is $\mathscr{F}(t)$ progressively measurable, and $E\left[\int_{0}^{\infty} \pi^{2}(t) X^{2}(t)\right]<\infty, p(t) \in[0,1], \pi(t) \in[0,+\infty) . \Lambda$ denotes the set of all admissible strategies $\Delta$ and (8) has a unique (strong) solution. Suppose the insurer has a utility function $U(x)$ which is strictly concave and continuously differentiable on $(-\infty,+\infty)$ and aims to maximize the expected utility of his/her terminal wealth.

For an admissible strategy $\Delta=(p(t), \pi(t))$, the value function $J^{\Delta}(t, x, r)$ from state $(x, r)$ at time $t$ is defined as

$$
J^{\Delta}(t, x, r)=E[U(X(T)) \mid X(t)=x, r(t)=r],
$$

and the objective function is

$$
H(t, x, r)=\max _{\Delta \in \Lambda} J^{\Delta}(t, x, r)
$$

with the boundary condition

$$
H(T, x, r)=U(x)
$$

The goal of the insurer is to find an optimal strategy $\Delta^{*}=$ $\left(p^{*}(t), \pi^{*}(t)\right)$ such that $J^{\Delta^{*}}(t, x, r)=H(t, x, r)$, where $p^{*}(t)$ is called the optimal reinsurance strategy and $\pi^{*}(t)$ is called the optimal investment strategy.

3.1. An Insurer with Reward Budget under the Vasicek Model. The stochastic interest rate followed by the Vasicek model is

$$
\mathrm{d} r_{t}=\left(\beta-\alpha r_{t}\right) \mathrm{d} t+\delta \mathrm{d} \widehat{W}(t)
$$


For mathematical treatment, there is no real difficulties besides representation of results being more complex when $u(t)$ is a positive real function. So we take $u(t)$ as a constant $u>0$ for the representation of results being more concise.

Theorem 1. For the optimal investment-reinsurance problem (15), assuming the utility is given by a power function $U(x)=$ $x^{q}, 0<q<1, T$ denoting the terminal time, then the optimal strategy $\Delta^{*}=\left(p^{*}, \pi^{*}\right)$ is given by

$$
\begin{aligned}
& p^{*}=-x \\
& \cdot \frac{(1-d) \eta \lambda \mu+\rho_{1} \delta \sqrt{\lambda} \sigma\left[-e^{-T \alpha}\left(-e^{t \alpha}+e^{T \alpha}\right)(-1+k) q / \alpha\right]}{\lambda \sigma^{2}(q-1)}, \\
& \pi^{*}=-\frac{u+\rho_{2} \delta v\left[-e^{-T \alpha}\left(-e^{t \alpha}+e^{T \alpha}\right)(-1+k) q / \alpha\right]}{v^{2}(1-k)(q-1)} .
\end{aligned}
$$

And the optimal value function is

$$
\begin{aligned}
H(t, x, r)= & e^{-e^{-2 T \alpha} q[A+B-C] / 2(-1+q) v^{2} \alpha^{2} \sigma^{2}} \\
& \cdot e^{e^{-2 T \alpha} q[E(t)+F(t)-G(t)] / 2(-1+q) v^{2} \alpha^{2} \sigma^{2}} \\
& \cdot e^{-\left(e^{-T \alpha}\left(-e^{t \alpha}+e^{T \alpha}\right)(-1+k) q / \alpha\right) r} x^{q},
\end{aligned}
$$

in which

$$
\begin{aligned}
& A=\frac{e^{2 T \alpha}(-1+k)^{2} q v^{2} \delta^{2}\left(1-q+q \rho_{1}^{2}+q \rho_{2}^{2}\right) \sigma^{2}}{2 \alpha}, \\
& B=e^{2 T \alpha} T\left[u^{2} \alpha^{2} \sigma^{2}-2(-1+k) q u v \alpha \delta \rho_{2} \sigma^{2}\right. \\
& \left.\quad+v^{2}\left(B_{1}+B_{2}+B_{3}\right)\right], \\
& C=\frac{2 e^{2 T \alpha}(-1+k) v \sigma\left[C_{1}+q C_{2}\right]}{\alpha}, \\
& E(t)=\frac{e^{2 t \alpha}(-1+k)^{2} q v^{2} \delta^{2}\left(1-q+q \rho_{1}^{2}+q \rho_{2}^{2}\right) \sigma^{2}}{2 \alpha}, \\
& F(t)=e^{2 T \alpha} t\left(u^{2} \alpha^{2} \sigma^{2}-2(-1+k) q u v \alpha \delta \rho_{2} \sigma^{2}\right. \\
& \left.\quad+v^{2}\left(F_{1}+F_{2}+F_{3}\right)\right),
\end{aligned}
$$

$$
=\frac{2 e^{t \alpha+T \alpha}(-1+k) v \sigma\left(-v \alpha \beta \sigma+G_{1}+q\left(-u \alpha \delta \rho_{2} \sigma+G_{2}\right)\right)}{\alpha},
$$

where $B_{1}, B_{2}, B_{3}, C_{1}, C_{2}, F_{1}, F_{2}, F_{3}, G_{1}$, and $G_{2}$ are given by

$$
\begin{aligned}
B_{1} & =(-1+k)^{2} q \delta^{2}\left(1+q\left(-1+\rho_{1}^{2}+\rho_{2}^{2}\right)\right) \sigma^{2}, \\
B_{2} & =\alpha^{2}\left((-1+d)^{2} \eta^{2} \lambda \mu^{2}+2 k(-1+q) \sigma^{2}\right), \\
B_{3} & =2(-1+k) \alpha \sigma\left(-\beta \sigma+q\left((-1+d) \delta \eta \sqrt{\lambda} \mu \rho_{1}\right.\right. \\
& +\beta \sigma)) \\
C_{1} & =-v \alpha \beta \sigma+(-1+k) q^{2} v \delta^{2}\left(-1+\rho_{1}^{2}+\rho_{2}^{2}\right) \sigma, \\
C_{2} & =\left(-u \alpha \delta \rho_{2} \sigma+v\left((-1+k) \delta^{2} \sigma\right.\right. \\
& \left.\left.+\alpha\left((-1+d) \delta \eta \sqrt{\lambda} \mu \rho_{1}+\beta \sigma\right)\right)\right) \\
F_{1} & =(-1+k)^{2} q \delta^{2}\left(1+q\left(-1+\rho_{1}^{2}+\rho_{2}^{2}\right)\right) \sigma^{2}, \\
F_{2} & =\alpha^{2}\left((-1+d)^{2} \eta^{2} \lambda \mu^{2}+2 k(-1+q) \sigma^{2}\right), \\
F_{3} & =2(-1+k) \alpha \sigma\left(-\beta \sigma+q\left((-1+d) \delta \eta \sqrt{\lambda} \mu \rho_{1}\right.\right. \\
& +\beta \sigma)), \\
G_{1} & =(-1+k) q^{2} v \delta^{2}\left(-1+\rho_{1}^{2}+\rho_{2}^{2}\right) \sigma, \\
G_{2} & =v\left((-1+k) \delta^{2} \sigma+\alpha\left((-1+d) \delta \eta \sqrt{\lambda} \mu \rho_{1}+\beta \sigma\right)\right)
\end{aligned}
$$

Proof. For any $H(t, x, r) \in C^{1,2,2}\left([0, T] \times R_{+} \times R_{+}\right)$, according to the classical results of Fleming and Soner [11], the generator can be defined as a variational operator:

$$
\mathscr{A} H^{\Delta}(t, x, r)=H_{t}+H_{x}[r(1-k) x-k x]+H_{r}(\beta
$$

$$
\begin{aligned}
& -\alpha r)+H_{r r} \frac{1}{2} \delta^{2}+\left\{H_{x}(1-d) p \eta \lambda \mu\right. \\
& \left.+H_{x r} \rho_{1} \delta p \sqrt{\lambda} \sigma+H_{x x} \frac{1}{2} p^{2} \lambda \sigma^{2}\right\} \\
& +\left\{H_{x} \pi u(t)(1-k) x+H_{x r} \rho_{2} \delta v \pi(1-k) x\right. \\
& \left.+H_{x x} \frac{1}{2} v^{2} \pi^{2}(1-k)^{2} x^{2}\right\},
\end{aligned}
$$

where $H_{t}, H_{x}, H_{r}, H_{x x}, H_{r r}$, and $H_{x r}$ denote the corresponding first- and second-order partial derivatives with respect to the corresponding variables $t, x, r$, respectively. The Hamilton-Jacobi-Bellman (HJB) equation for problem (15) is given by

$$
\sup _{\Delta \in \Lambda}\left\{\mathscr{A} H^{\Delta}(t, x, r)\right\}=0
$$

with the boundary condition $H(T, x, r)=x^{q}$.

According to the first-order necessary condition for the extreme, the following equation is obtained from (23):

$$
\begin{aligned}
& H_{x}(1-d) \eta \lambda \mu+H_{x r} \rho_{1} \delta \sqrt{\lambda} \sigma+H_{x x} p \lambda \sigma^{2}=0, \\
& H_{x} u(1-k) x+H_{x r} \rho_{2} \delta v(1-k) x \\
& \quad+H_{x x} v^{2} \pi(1-k)^{2} x^{2}=0 .
\end{aligned}
$$


The solution of the above equation gives the optimal feedback control:

$$
\begin{aligned}
& p^{*}=-\frac{H_{x}(1-d) \eta \lambda \mu+H_{x r} \rho_{1} \delta \sqrt{\lambda} \sigma}{H_{x x} \lambda \sigma^{2}}, \\
& \pi^{*}=-\frac{H_{x} u+H_{x r} \rho_{2} \delta v}{H_{x x} v^{2}(1-k) x} .
\end{aligned}
$$

Plugging (25) into (23),

$$
\begin{aligned}
H_{t}+ & H_{x}[r(1-k) x-k x]+H_{r}(\beta-\alpha r)+H_{r r} \frac{1}{2} \delta^{2} \\
& -\frac{\left(H_{x}(1-d) \eta \sqrt{\lambda} \mu+H_{x r} \rho_{1} \delta \sigma\right)^{2}}{2 H_{x x} \sigma^{2}} \\
- & \frac{\left(H_{x} u+H_{x r} \rho_{2} \delta v\right)^{2}}{2 v^{2} H_{x x}}=0 .
\end{aligned}
$$

Conjecture of the solution has the following form:

$$
\begin{aligned}
H(t, x, r) & =f(t) e^{h(t) r} x^{q}, \\
H(T, x, r) & =x^{q}, \quad 0<q<1, \\
f(T) & =1, \\
h(T) & =0 .
\end{aligned}
$$

The derivatives of the conjecture with respect to $t, x, r$, respectively, are

$$
\begin{aligned}
H_{t} & =\left(f^{\prime}(t)+f(t) h^{\prime}(t) r\right) e^{h(t) r} x^{q}, \\
H_{r} & =f(t) e^{h(t) r} x^{q} h(t), \\
H_{r r} & =f(t) e^{h(t) r} x^{q} h(t)^{2}, \\
H_{x} & =f(t) e^{h(t) r} q x^{(q-1)}, \\
H_{x x} & =f(t) e^{h(t) r} q(q-1) x^{(q-2)}, \\
H_{x r} & =f(t) e^{h(t) r} q x^{(q-1)} h(t) .
\end{aligned}
$$

Plugging these derivatives into (26),

$$
\begin{aligned}
f^{\prime}(t) & +f(t) h^{\prime}(t) r+f(t) q[r(1-k)-k] \\
& +f(t) h(t)(\beta-\alpha r)+f(t) h(t)^{2} \frac{1}{2} \delta^{2} \\
& -\frac{\left((1-d) \eta \sqrt{\lambda} \mu+h(t) \rho_{1} \delta \sigma\right)^{2} f(t) q}{2(q-1) \sigma^{2}} \\
& -\frac{\left(u+h(t) \rho_{2} \delta v\right)^{2} f(t) q}{2(q-1) v^{2}}=0 .
\end{aligned}
$$

Splitting (29) into two equations,

$$
\begin{aligned}
& h^{\prime}(t)-\alpha h(t)+q(1-k)=0, \\
& h(T)=0, \\
& \frac{f^{\prime}(t)}{f(t)}=k q-h(t) \beta-\frac{1}{2} \delta^{2} h(t)^{2} \\
& +\left[\frac{\left((1-d) \eta \sqrt{\lambda} \mu+h(t) \rho_{1} \delta \sigma\right)^{2}}{2(q-1) \sigma^{2}}\right. \\
& \left.+\frac{\left(u+h(t) \rho_{2} \delta v\right)^{2}}{2(q-1) v^{2}}\right] q=0, \\
& f(T)=1 .
\end{aligned}
$$

Solving the above two ordinary differential equations, the solutions $h(t)$ and $f(t)$ are given by

$$
\begin{aligned}
& h(t)=-\frac{e^{-T \alpha}\left(-e^{t \alpha}+e^{T \alpha}\right)(-1+k) q}{\alpha} \\
& f(t)=e^{-e^{-2 T \alpha} q[A+B-C] / 2(-1+q) v^{2} \alpha^{2} \sigma^{2}}
\end{aligned}
$$

$$
\cdot e^{e^{-2 T \alpha} q[E(t)+F(t)-G(t)] / 2(-1+q) v^{2} \alpha^{2} \sigma^{2},}
$$

where

$$
\begin{aligned}
& A= \frac{e^{2 T \alpha}(-1+k)^{2} q v^{2} \delta^{2}\left(1-q+q \rho_{1}^{2}+q \rho_{2}^{2}\right) \sigma^{2}}{2 \alpha}, \\
& B= e^{2 T \alpha} T\left[u^{2} \alpha^{2} \sigma^{2}-2(-1+k) q u v \alpha \delta \rho_{2} \sigma^{2}\right. \\
&\left.+v^{2}\left(B_{1}+B_{2}+B_{3}\right)\right], \\
& C= \frac{2 e^{2 T \alpha}(-1+k) v \sigma\left[C_{1}+q C_{2}\right]}{\alpha}, \\
& E(t)=\frac{e^{2 t \alpha}(-1+k)^{2} q v^{2} \delta^{2}\left(1-q+q \rho_{1}^{2}+q \rho_{2}^{2}\right) \sigma^{2}}{2 \alpha}, \\
& F(t)=e^{2 T \alpha} t\left(u^{2} \alpha^{2} \sigma^{2}-2(-1+k) q u v \alpha \delta \rho_{2} \sigma^{2}\right. \\
&+\left.v^{2}\left(F_{1}+F_{2}+F_{3}\right)\right), \\
& G(t) \\
&=\frac{2 e^{t \alpha+T \alpha}(-1+k) v \sigma\left(-v \alpha \beta \sigma+G_{1}+q\left(-u \alpha \delta \rho_{2} \sigma+G_{2}\right)\right)}{\alpha}
\end{aligned}
$$


and in which $B_{1}, B_{2}, B_{3}, C_{1}, C_{2}, F_{1}, F_{2}, F_{3}, G_{1}$, and $G_{2}$ are given by

$$
\begin{aligned}
B_{1} & =(-1+k)^{2} q \delta^{2}\left(1+q\left(-1+\rho_{1}^{2}+\rho_{2}^{2}\right)\right) \sigma^{2}, \\
B_{2} & =\alpha^{2}\left((-1+d)^{2} \eta^{2} \lambda \mu^{2}+2 k(-1+q) \sigma^{2}\right), \\
B_{3} & =2(-1+k) \alpha \sigma\left(-\beta \sigma+q\left((-1+d) \delta \eta \sqrt{\lambda} \mu \rho_{1}\right.\right. \\
& +\beta \sigma)), \\
C_{1} & =-v \alpha \beta \sigma+(-1+k) q^{2} v \delta^{2}\left(-1+\rho_{1}^{2}+\rho_{2}^{2}\right) \sigma, \\
C_{2} & =\left(-u \alpha \delta \rho_{2} \sigma+v\left((-1+k) \delta^{2} \sigma\right.\right. \\
& \left.\left.+\alpha\left((-1+d) \delta \eta \sqrt{\lambda} \mu \rho_{1}+\beta \sigma\right)\right)\right), \\
F_{1} & =(-1+k)^{2} q \delta^{2}\left(1+q\left(-1+\rho_{1}^{2}+\rho_{2}^{2}\right)\right) \sigma^{2}, \\
F_{2} & =\alpha^{2}\left((-1+d)^{2} \eta^{2} \lambda \mu^{2}+2 k(-1+q) \sigma^{2}\right), \\
G_{1} & =(-1+k) q^{2} v \delta^{2}\left(-1+\rho_{1}^{2}+\rho_{2}^{2}\right) \sigma, \\
F_{3} & =2(-1+k) \alpha \sigma\left(-\beta \sigma+q\left((-1+d) \delta \eta \sqrt{\lambda} \mu \rho_{1}\right.\right. \\
& +\beta \sigma)), \\
G_{1} &
\end{aligned}
$$

Plugging (31) into (27), the optimal value function is obtained as follows:

$$
\begin{aligned}
H(t, x, r)= & e^{-e^{-2 T \alpha} q[A+B-C] / 2(-1+q) v^{2} \alpha^{2} \sigma^{2}} \\
& \cdot e^{e^{-2 T \alpha} q[E(t)+F(t)-G(t)] / 2(-1+q) v^{2} \alpha^{2} \sigma^{2}} \\
& \cdot e^{-\left(e^{-T \alpha}\left(-e^{t \alpha}+e^{T \alpha}\right)(-1+k) q / \alpha\right) r} x^{q} .
\end{aligned}
$$

Then the corresponding derivatives being plugged into (25), the optimal strategies $\Delta^{*}$ are given by

$$
\begin{aligned}
p^{*} & =-x \\
\cdot & \frac{(1-d) \eta \lambda \mu+\rho_{1} \delta \sqrt{\lambda} \sigma\left[-e^{-T \alpha}\left(-e^{t \alpha}+e^{T \alpha}\right)(-1+k) q / \alpha\right]}{\lambda \sigma^{2}(q-1)}, \\
\pi^{*} & =-\frac{u+\rho_{2} \delta v\left[-e^{-T \alpha}\left(-e^{t \alpha}+e^{T \alpha}\right)(-1+k) q / \alpha\right]}{v^{2}(1-k)(q-1)} .
\end{aligned}
$$

Theorem 2 (verification theorem). Let $H(t, x, r)$ be a convex, twice differential solution to (23) with the boundary condition (16) such that $\left|H\left(0, x_{0}, r_{0}\right)\right|<\infty$. Then, for all $t \in[0, T], x \in$ $(0, \infty)$, (i) $H(t, x, r) \leq J^{\Delta}(t, x, r)$ for every admissible strategy $\Delta \epsilon$ $\Lambda$.

(ii) If there exists an admissible control $\Delta^{*}=\left(p^{*}, \pi^{*}\right)$ such that

$$
\Delta^{*} \in \operatorname{argmax}\left[\mathscr{A}^{\Delta} H\left(t, x^{*}(t), r^{*}(t)\right)\right]
$$

then the value function $H(t, x, r)=J^{\Delta^{*}}(t, x, r)$ and the policy $\Delta^{*}=\left(p^{*}, \pi^{*}\right)$ is the optimal strategy corresponding to $X^{\Delta^{*}}(t)$ which is the solution of (8).

Proof. The proof is classical; refer to Fleming and Soner [11], Guan and Liang $[12,13]$, Gu et al. [14], and so on; we omit it here.

3.2. A Special Case of an Insurer with Reward Budget under a Constant Interest Rate. In this subsection, we investigate a special case that the interest rate $r(t)$ is a constant $r$. All assumptions are the same as above except that $r(t) \equiv r$ throughout this part; thus, we have

$$
\begin{aligned}
& \frac{\mathrm{d} S^{0}(t)}{S^{0}(t)}=r \mathrm{~d} t, \quad S^{0}(0)=1, \\
& \frac{\mathrm{d} S(t)}{S(t)}=[r+u(t)] \mathrm{d} t+v \mathrm{~d} \widetilde{W}(t), \quad S(0)=s_{0},
\end{aligned}
$$

where $r, v$ are positive constants and $u(t)$ is a positive realvalued function.

The surplus processes with claim and reinsurance are the same as (5) and (6), and $W(t)$ and $\widetilde{W}(t)$ are independent standard Brownian motions. So we get the wealth process with reward budget, investing in the financial market as follows:

$$
\begin{aligned}
& \mathrm{d} X(t)=[(1-k) X(t) r-k X(t)+(1-d) \eta p \lambda \mu \\
& \quad+\pi(t)(1-k) X(t) u(t)] \mathrm{d} t+\pi(t)(1-k) X(t) \\
& \quad \cdot v \mathrm{~d} \widetilde{W}(t)+p \sqrt{\lambda} \sigma \mathrm{d} W(t) .
\end{aligned}
$$

We consider that the utility $U(x)$ is a power utility (constant relative risk aversion) function

$$
U(x)=x^{q}
$$

to maximize the expected utility of terminal wealth, where the constant $q \in(0,1)$ is the relative risk aversion coefficient of a risk averse insurer. For an admissible strategy $\Delta=$ $(p(t), \pi(t))$, the value function $J^{\Delta}(t, x, r)$ from state $x$ at time $t$ is defined as

$$
J^{\Delta}(t, x)=E[U(X(T)) \mid X(t)=x],
$$

and the optimal value function is given by

$$
H(t, x)=\max _{(\pi, p)} J^{\Delta}(t, x)
$$

with the boundary condition $H(T, x, r)=x^{q}$. 
Applying the classical tools of stochastic optimal control, if the optimal value function $H(t, x) \in C^{1,2}([0, T] \times R)$, then $H$ satisfies the following Hamilton-Jacobi-Bellman (HJB) equation:

$$
\begin{aligned}
& H_{t}+H_{x}[r-(1+r) k] x \\
& +\max _{p}\left\{H_{x}(1-d) p \eta \lambda \mu+H_{x x} \frac{1}{2} p^{2} \lambda \sigma^{2}\right\} \\
& +\max _{\pi}\left\{H_{x} \pi u(t)(1-k) x+H_{x x} \frac{1}{2} v^{2} \pi^{2}(1-k)^{2} x^{2}\right\} \\
& =0 .
\end{aligned}
$$

According to the first-order necessary condition of extreme, we obtain that

$$
\begin{aligned}
p^{*} & =-\frac{(1-d) \eta \mu H_{x}}{\sigma^{2} H_{x x}}, \\
\pi^{*} & =-\frac{u(t) H_{x}}{v^{2}(1-k) x H_{x x}} .
\end{aligned}
$$

Conjecture of the solution has the following form:

$$
\begin{aligned}
H(t, x) & =f(t) x^{q}, \\
H(T, x) & =x^{q}, \quad 0<q<1, \\
f(T) & =1 .
\end{aligned}
$$

The derivatives of the conjecture with respect to $t, x$, respectively, are

$$
\begin{aligned}
H_{t} & =f^{\prime}(t) x^{q}, \\
H_{x} & =f(t) q x^{(q-1)}, \\
H_{x x} & =f(t) q(q-1) x^{(q-2)} .
\end{aligned}
$$

Plugging these derivatives into the HJB equation, we have

$$
\begin{aligned}
f^{\prime}(t) & +f(t) q[r-(1+r) k]+\frac{[(1-d) \eta \mu]^{2} \lambda q f(t)}{2 \sigma^{2}(1-q)} \\
& +\frac{[u(t)]^{2} q f(t)}{2 v^{2}(1-q)}=0 .
\end{aligned}
$$

The solution of the above ordinary differential equation is

$$
\begin{aligned}
& f(t)=\exp \left\{q \int_{t}^{T}\left[r-(1+r) k+\frac{(1-d)^{2} \eta^{2} \lambda \mu^{2}}{2 \sigma^{2}(1-q)}+\frac{u(\tau)^{2}}{2 v^{2}(1-q)}\right]\right. \\
& \mathrm{d} \tau\} .
\end{aligned}
$$

We obtain the value function

$$
\begin{aligned}
& H(t, x)=x^{q} \\
& \quad \cdot \exp \left\{\left[r-(1+r) k+\frac{(1-d)^{2} \eta^{2} \lambda \mu^{2}}{2 \sigma^{2}(1-q)}\right] q(T-t)\right. \\
& \left.\quad+q \int_{t}^{T}\left[\frac{u(\tau)^{2}}{2 v^{2}(1-q)}\right] \mathrm{d} \tau\right\}
\end{aligned}
$$

and the optimal strategy $\alpha^{*}=\left(p^{*}, \pi^{*}\right)$ as follows:

$$
\begin{aligned}
p^{*} & =\frac{1-d}{(1-q)} \cdot \frac{\eta \mu x}{\sigma^{2}}, \\
\pi^{*} & =\frac{1}{(1-k)(1-q)} \cdot \frac{u(t)}{v^{2}} .
\end{aligned}
$$

For simplicity, if the positive real-valued function $u(t)$ taken as a positive constant $u>0$, it does not bring substantial impact on the problem investigated. We obtain the optimal value function

$$
\begin{aligned}
& H(t, x)=x^{q} \exp \left\{\left[r-(1+r) k+\frac{(1-d)^{2} \eta^{2} \lambda \mu^{2}}{2 \sigma^{2}(1-q)}\right.\right. \\
& \left.\left.+\frac{u^{2}}{2 v^{2}(1-q)}\right] q(T-t)\right\}
\end{aligned}
$$

and the optimal strategy $\alpha^{*}=\left(p^{*}, \pi^{*}\right)$ as follows:

$$
\begin{aligned}
p^{*} & =\frac{(1-d) \eta \mu x}{(1-q) \sigma^{2}}, \\
\pi^{*} & =\frac{u}{v^{2}(1-k)(1-q)} .
\end{aligned}
$$

3.3. Some Economic Interpretations. The optimal strategy $\Delta^{*}=\left(p^{*}, \pi^{*}\right)$ under a constant interest rate is

$$
\begin{aligned}
& \left(p^{*}, \pi^{*}\right) \\
& \quad=\left(\frac{x}{(1-q)} \cdot \frac{(1-d) \eta \mu}{\sigma^{2}}, \frac{1}{(1-k)(1-q)} \cdot \frac{u}{v^{2}}\right)
\end{aligned}
$$

and the optimal strategy $\Delta^{*}=\left(p^{*}, \pi^{*}\right)$ under the stochastic interest rates is

$$
\begin{aligned}
& \left(p^{*}, \pi^{*}\right)=(x \\
& \cdot \frac{(1-d) \eta \lambda \mu+\rho_{1} \delta \sqrt{\lambda} \sigma\left[e^{-T \alpha}\left(-e^{t \alpha}+e^{T \alpha}\right)(1-k) q / \alpha\right]}{\lambda \sigma^{2}(1-q)}, \\
& \left.\frac{u+\rho_{2} \delta v\left[e^{-T \alpha}\left(-e^{t \alpha}+e^{T \alpha}\right)(1-k) q / \alpha\right]}{v^{2}(1-k)(1-q)}\right) .
\end{aligned}
$$


In this subsection, the results obtained under constant interest rate will serve as a reference. So as to comprehend the implicit economic meaning of abstract formula more clearly, we will make the following interpretations.

Remark 3. Just looking at the optimal strategy under the constant interest rate, it is not difficult to give the following economic interpretations.

First, the optimal reinsurance proportion

$$
p^{*}=\frac{x}{(1-q)} \cdot \frac{(1-d) \eta \mu}{\sigma^{2}}=\frac{1}{(1-q) / x} \cdot \frac{(1-d) \eta \mu}{\sigma^{2}}
$$

is determined by two key factors $(1-q) / x$ and $(1-d) \eta \mu / \sigma^{2}$.

Equation (11) shows that $(1-q) / x$ is the coefficient of absolute risk aversion, which gives a measurable indicator of an insurer's absolute risk aversion. So the expression $1 /((1-$ $q) / x$ ) represents the risk tolerance coefficient and the optimal reinsurance proportion $p^{*}$ is in direct proportion to the risk tolerance coefficient $1 /((1-q) / x)$. Intuitive understanding is that there would be greater risk scale in the insurer's businesses if an insurer has greater risk tolerance (the risk tolerance coefficient is bigger), so that the insurance company having larger size of risk needs to be transferred to the reinsurance companies.

As for this expression $(1-d) \eta \mu / \sigma^{2}$, it represents the rate of underwriting return. An insurer assumes a certain claim risk $\sigma^{2}$, the higher rate of underwriting return which would be more advantageous to the insurance company.

Second, the optimal investment proportion

$$
\pi^{*}=\frac{1}{(1-q)} \cdot \frac{1}{(1-k)} \cdot \frac{u}{v^{2}}
$$

is also based on three key factors $(1-q),(1-k)$, and $u / v^{2}$.

Equation (13) shows that $(1-q)$ is the coefficient of relative risk aversion, which gives a measurable indicator of an insurer's relative risk aversion. So the expression $1 /(1-$ q) represents the relative risk tolerance coefficient and the optimal investment proportion $\pi^{*}$ is in direct proportion to the relative risk tolerance coefficient $1 /(1-q)$. This is very natural, because the companies of high risk tolerance always prefer risk assets investment for that they is more valued high yield hidden in high risk.

As for the expression $u / v^{2}=((r+u)-r) / v^{2}$, it is similar to the market price of risk in financial market. $(1-k)$ represents the total capital share an insurer can use to invest. The amount of risk investment is restricted by the insurer's risk tolerance, so the proportion of risk investment seems smaller if the total capital share is greater.

Remark 4. Comparing the results of constant interest rate with the results of stochastic interest rate, there is one more item $x \cdot\left(\rho_{1} \delta / \sqrt{\lambda} \sigma\right) \cdot\left[e^{-T \alpha}\left(-e^{t \alpha}+e^{T \alpha}\right)(1-k) q\right] /(1-q) \alpha$ of the optimal reinsurance proportion under the stochastic interest rate than the optimal reinsurance proportion under a constant interest rate:

$$
\begin{aligned}
& p^{*} \text { stochastic }-p^{*} \text { constant } \\
& \quad=\frac{q}{(1-q)} \cdot \frac{\rho_{1} \delta}{\sqrt{\lambda} \sigma} \cdot \frac{\left[\left(1-e^{-(T-t) \alpha}\right)\right]}{\alpha}(1-k) x .
\end{aligned}
$$

If $\rho_{1}>0$, then $p^{*}$ stochastic $>p^{*}$ constant; else if $\rho_{1}<0$, then $p^{*}$ stochastic $<p^{*}$ constant.

In fact, $\left[\left(1-e^{-(T-t) \alpha}\right)\right] / \alpha$ is the changing expectations of the interest rate described by a Vasicek model under the risk neutral probability measure. So $\left(\left[\left(1-e^{-(T-t) \alpha}\right)\right] / \alpha\right)(1-k) x$ reflects the change of insurance company's wealth with time $t$. $q /(1-q)$ is the characterization of risk aversion of an insurer. $\rho_{1} \delta / \sqrt{\lambda} \sigma$ is a ratio of interest rate volatility rate divided by the volatility rate of claim size. It is not hard to see the crucial effects determined by the correlation of the interest rate fluctuations and the volatility of claim amount.

Similarly, there is one more item $\left(\rho_{2} \delta / v\right) \cdot\left(\left[e^{-T \alpha}\left(-e^{t \alpha}+\right.\right.\right.$ $\left.\left.\left.e^{T \alpha}\right) q\right] /(1-q) \alpha\right)$ of the optimal investment proportion under the stochastic interest rate than the optimal investment proportion under a constant interest rate:

$$
\pi^{*} \text { stochastic }-\pi^{*} \text { constant }
$$

$$
=\frac{q}{(1-q)} \cdot \frac{\rho_{2} \delta}{v} \cdot \frac{\left[\left(1-e^{-(T-t) \alpha}\right)\right]}{\alpha} .
$$

If $\rho_{2}>0$, then $\pi^{*}$ stochastic $>\pi^{*}$ constant; else if $\rho_{2}<0$, then $\pi^{*}$ stochastic $<\pi^{*}$ constant.

Same as the above, $\left[\left(1-e^{-(T-t) \alpha}\right)\right] / \alpha$ is the changing expectations of the interest rate described by a Vasicek model under the risk neutral probability measure. $q /(1-q)$ is the characterization of risk aversion of an insurer. But $\delta / v$ is a ratio of interest rate volatility rate divided by the volatility rate of risky assets, so that the crucial effects are determined by the correlation of the interest rate fluctuations and the risk assets volatility.

In conclusion, the primary differences from the optimal strategy under a constant interest rate are implicit in the two expressions $x \cdot\left(\rho_{1} \delta / \sqrt{\lambda} \sigma\right) \cdot\left(\left[e^{-T \alpha}\left(-e^{t \alpha}+e^{T \alpha}\right)(1-k) q\right] /(1-q) \alpha\right)$ and $\left(\rho_{2} \delta / v\right) \cdot\left(\left[e^{-T \alpha}\left(-e^{t \alpha}+e^{T \alpha}\right) q\right] /(1-q) \alpha\right)$, which reflect the correlation of the stochastic interest rate and the volatility of claim amount and the correlation of the stochastic interest rate and the risk assets volatility, respectively. However, this kind of distinction is a synergy effect based on interest rate volatility, risky asset volatility, claim size volatility, investor's risk aversion, and so forth. 
Remark 5. In the last part of this subsection, let us focus on the actual economic meaning of the optimal value function. The optimal value function under a constant interest rate is

$$
\begin{aligned}
& H(t, x)=x^{q} \exp \left\{\left[r-(1+r) k+\frac{(1-d)^{2} \eta^{2} \lambda \mu^{2}}{2 \sigma^{2}(1-q)}\right.\right. \\
& \left.\left.+\frac{u^{2}}{2 v^{2}(1-q)}\right] q(T-t)\right\}=x^{q} \frac{e^{(1-k) r q(T-t)}}{e^{-k q(T-t)}} \\
& \quad \cdot \exp \left\{\left[\frac{(1-d)^{2} \eta^{2} \lambda \mu^{2}}{2 \sigma^{2}(1-q)}+\frac{u^{2}}{2 v^{2}(1-q)}\right] q(T-t)\right\}
\end{aligned}
$$

and the optimal value function under the stochastic interest rate is

$$
\begin{aligned}
H(t, x, r)= & x^{q} \cdot e^{-e^{-2 T \alpha} q[A+B-C] / 2(-1+q) v^{2} \alpha^{2} \sigma^{2}} \\
& \cdot e^{e^{-2 T \alpha} q[E(t)+F(t)-G(t)] / 2(-1+q) v^{2} \alpha^{2} \sigma^{2}} \\
& \cdot e^{-\left(e^{-T \alpha}\left(-e^{t \alpha}+e^{T \alpha}\right)(-1+k) q / \alpha\right) r} \\
= & x^{q} \cdot e^{-e^{-2 T \alpha} q[A+B-C] / 2(-1+q) v^{2} \alpha^{2} \sigma^{2}} \\
& \cdot e^{e^{-2 T \alpha} q[E(t)+F(t)-G(t)] / 2(-1+q) v^{2} \alpha^{2} \sigma^{2}} \\
& \cdot \frac{e^{\left(\left(1-e^{-(T-t) \alpha}\right) / \alpha\right) r q}}{e^{\left(\left(1-e^{-(T-t) \alpha}\right) / \alpha\right) r k q} .}
\end{aligned}
$$

The optimal value function represents the maximum expected utility of wealth, which increases with the wealth increasing. The key factors of wealth changes mainly depend on the following four aspects: the return of risk-free investment, the share of reward budget, the rate of underwriting return, and the yields rate of risk investment. For the optimal value function under a constant interest rate, $e^{-(t-T)(1-k) r q}$ represents the impact on insurer's utility of the discounted value at time $t, e^{-k q(T-t)}$ indicates the loss of utility caused by the share $k$ of reward budget, $\left((1-d)^{2} \eta^{2} \lambda \mu^{2} / 2 \sigma^{2}(1-q)\right) q(T-t)$ denotes the rate of underwriting return for the risk volatility $\sigma^{2}$ in insurance business, and $\left(u^{2} / 2 v^{2}(1-q)\right) q(T-t)$ signifies the yields rate of risk investment for the risk volatility $v^{2}$. It is worth noting that the risk aversion factor $q$ is included in each expression, because that the utility itself is closely related with risk preference.

For the optimal value function under the stochastic interest rate, the key factors of wealth changes mainly depend on the following aspects: the stochastic interest rate, the share of reward budget, the rate of underwriting return, and the yields rate of risk investment. We can give the similar explanations by reference to economic meanings under the constant interest rate. For example, $e^{\left(e^{-T \alpha}\left(-e^{t \alpha}+e^{T \alpha}\right) q / \alpha\right) r}, e^{\left(e^{-T \alpha}\left(-e^{t \alpha}+e^{T \alpha}\right) k q / \alpha\right) r}$ reflect the action effects of the stochastic interest rate and the share of reward budget, respectively. $e^{-e^{-2 T \alpha} q[A+B-C] / 2(-1+q) v^{2} \alpha^{2} \sigma^{2}}$. $e^{e^{-2 T \alpha}} q[E(t)+F(t)-G(t)] / 2(-1+q) v^{2} \alpha^{2} \sigma^{2}$ denotes the comprehensive effect of the rate of underwriting return and the yields rate of risk investment. But it is hard to entirely separate the influence of one factor apart from the influence of other factors. Arithmetically, a combination of a variety of random factors appears in one expression in a complex way. It is noteworthy that the risk aversion factor $q$ is an important factor of an insurer's utility.

\section{Numerical Simulations}

In this section, we give some qualitative results to show the relationship between the optimal control strategies (optimal investment strategy and optimal proportional reinsurance strategy, resp.) and the parameters in our model. Unless otherwise stated, the hypothetical values of model parameters are as follows:

$$
\begin{aligned}
& x=3, \\
& \eta=0.1, \\
& \lambda=2, \\
& \mu=0.2, \\
& b=0.05, \\
& v=1, \\
& k=0.1, \\
& T=10, \\
& \delta=0.5, \\
& \sigma=2, \\
& \alpha=5, \\
& \beta=0.1, \\
& \rho_{1}=0.3, \\
& \rho_{2}=0.2, \\
& r=0.08, \\
& q=0.35, \\
& u=0.15, \\
& d=0.3 .
\end{aligned}
$$

The parameter $u$ is a risk premium which shows the excess return when the risk-free interest rate $r$ is subtracted from the expected return of risk assets. $\sigma, \delta, v$ are volatility parameters, indicating the risk volatility of claim size, interest rate, or risk assets, respectively. $\rho_{1}$ plays an important role in risk interconnection between interest rate and claim size. $\rho_{2}$ plays an important role in risk collaboration between interest rate and risk asset. Such risk correlations need more special attention when you make decisions.

It is known that the value function is the optimal expected utility of insurer' wealth when the insurer takes the optimal 


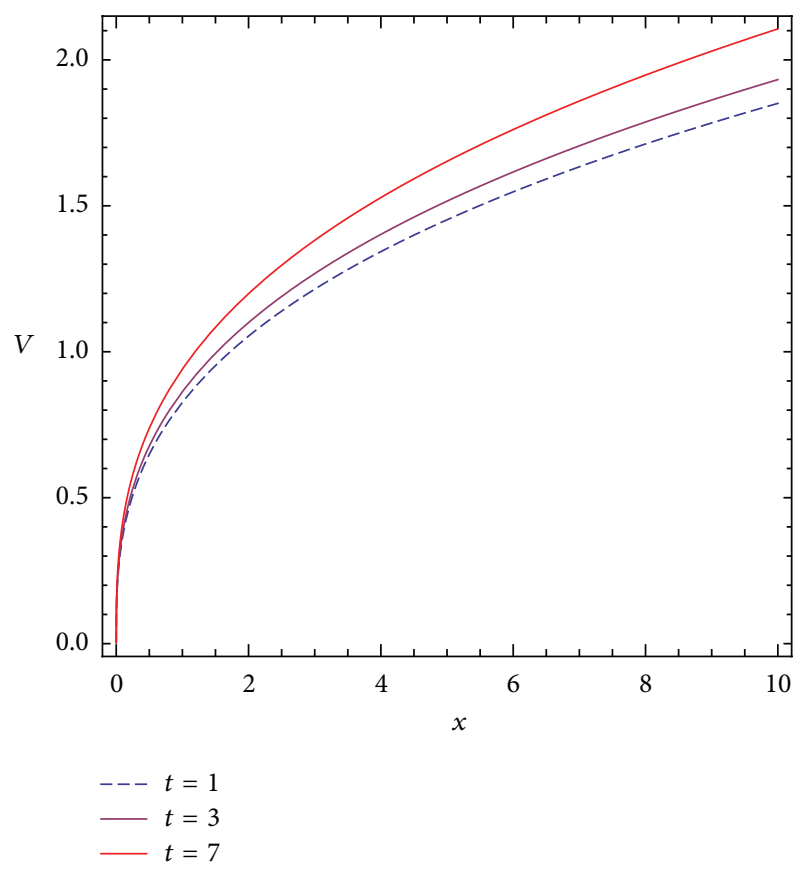

FIGURE 1: The optimal value function varies with respect to $x$.

strategy. First, let us pay attention to the optimal value function; see Figures 1, 2, 3, and 4:

$$
\begin{aligned}
H(t, x, r)= & e^{-e^{-2 T \alpha} q[A+B-C] / 2(-1+q) v^{2} \alpha^{2} \sigma^{2}} \\
& \cdot e^{e^{-2 T \alpha} q[E(t)+F(t)-G(t)] / 2(-1+q) v^{2} \alpha^{2} \sigma^{2}} \\
& \cdot e^{-\left(e^{-T \alpha}\left(-e^{t \alpha}+e^{T \alpha}\right)(-1+k) q / \alpha\right) r} x^{q} .
\end{aligned}
$$

In the case of other parameters being fixed, the influence of interest rate on the value function is not obvious, only when interest rates were significantly higher than normal value which can lead to a significant change in the value function. From the numerical illustration, we also can see that the growth trend of value function with the increase of interest rates will not appear within a short time period. Because the profit of investment on risk-free asset is so meager that it needs such a long enough time to show its observable growth trend.

With interest rates at a certain level, the risk premium $u$ of risk investment is not too much higher than the interest rate, and the impact of risk investment income on the value function is not obvious. Only when the risk investment income is too much higher than the interest rate, the influence of risk investment income on the utility is significant, which can be seen from the impact on the value function. In addition, the long-term observation and short-term observation still have big differences.

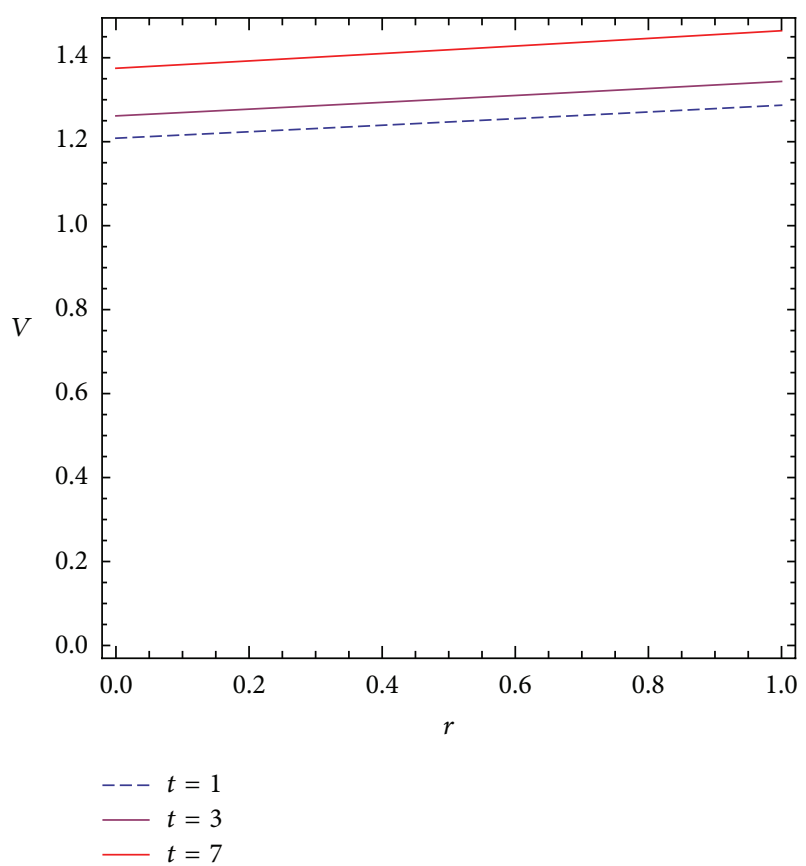

FIGURE 2: The optimal value function varies with respect to $r$.

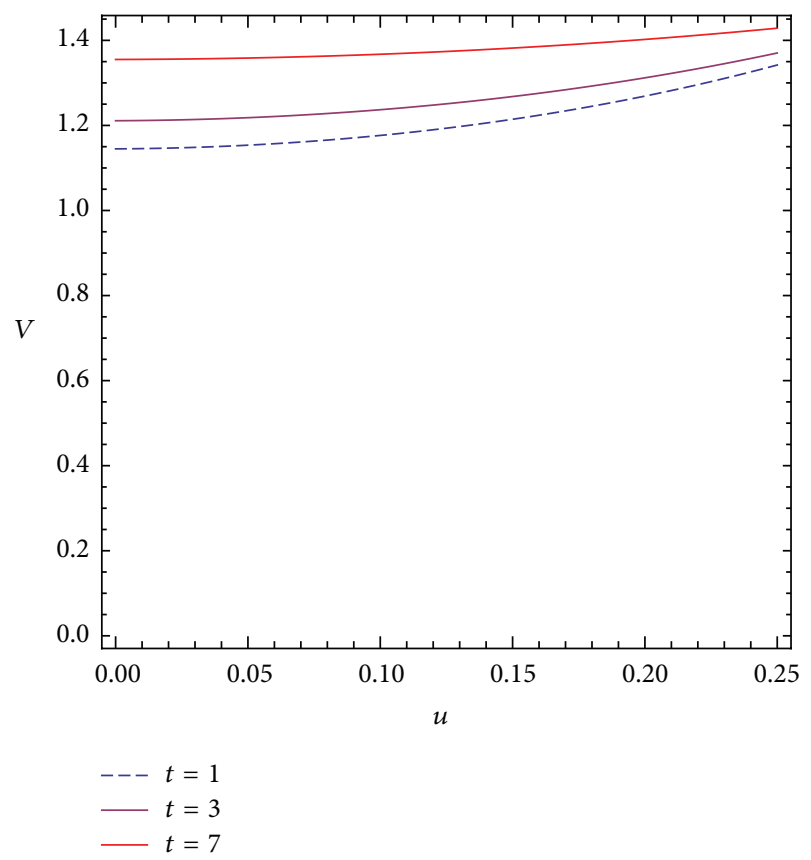

FIGURE 3: The optimal value function varies with respect to $u$ at low level.

Next, we show the variation trend of optimal reinsurance proportion:

$$
\begin{aligned}
p^{*} & =x\left[\frac{(1-d) \eta \mu}{\sigma^{2}(1-q)}\right. \\
& \left.+\frac{\rho_{1} \delta\left(e^{-T \alpha}\left(-e^{t \alpha}+e^{T \alpha}\right)(1-k) q / \alpha\right)}{\sqrt{\lambda} \sigma(1-q)}\right]
\end{aligned}
$$




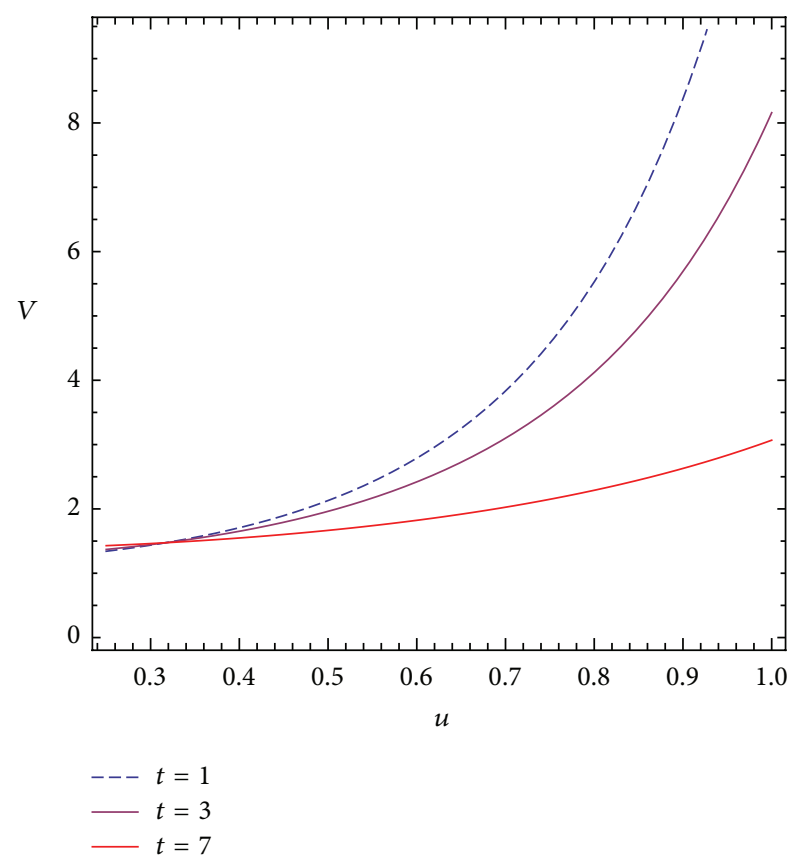

FIGURE 4: The optimal value function varies with respect to $u$ at high level.

$$
\begin{aligned}
= & x\left[\frac{\eta \mu}{\sigma^{2}} \cdot \frac{(1-d)}{(1-q)}+\rho_{1} \cdot \frac{\delta}{\sqrt{\lambda} \sigma}\right. \\
& \left.\cdot \frac{e^{-T \alpha}\left(-e^{t \alpha}+e^{T \alpha}\right)(1-k)}{\alpha} \frac{q}{(1-q)}\right]
\end{aligned}
$$

with respect to several key parameters $x, \eta$, $\mu$. See Figures 5 and 6.

As we can see from the expression of optimal reinsurance proportion $p^{*}$ immediately, it is mainly influenced by the average claim size $\lambda \mu$, the standard deviation of claim volatility $\sqrt{\lambda} \sigma$, and the variance of claim size $\sigma^{2}$. Based on the possible influence of the interest rate fluctuation on the volatility of claim size, the volatility of interest rate $\delta$, and correlation coefficient $\rho_{1}$ are also risk factors that appeared in the optimal reinsurance proportion strategy.

At last, the variation tendency of optimal investment strategy with respect to $u, v$

$$
\begin{aligned}
\pi^{*}= & \frac{u+\rho_{2} \delta v\left(e^{-T \alpha}\left(-e^{t \alpha}+e^{T \alpha}\right)(1-k) q / \alpha\right)}{v^{2}(1-k)(1-q)} \\
= & {\left[\frac{u}{v^{2}}+\rho_{2} \cdot \frac{\delta}{v} \frac{e^{-T \alpha}\left(-e^{t \alpha}+e^{T \alpha}\right)(1-k) q}{\alpha}\right] } \\
& \cdot \frac{1}{(1-k)(1-q)}
\end{aligned}
$$

will be illustrated; see Figures 7 and 8 .

With the return of risk investment being more higher than the interest rate, the share of investment on risk

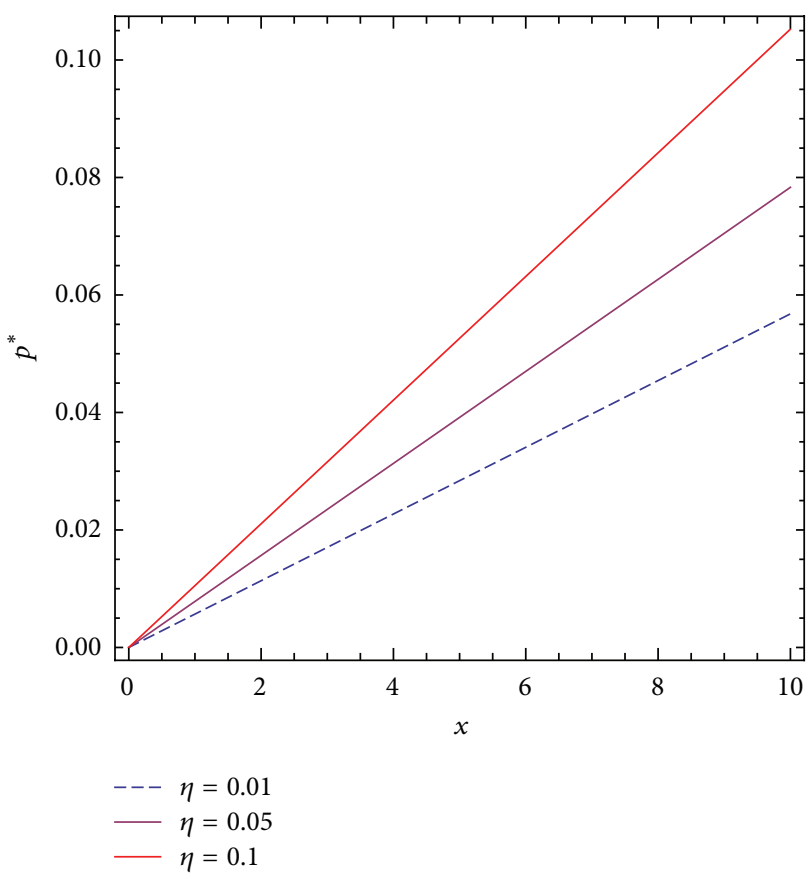

FIGURE 5: The optimal reinsurance proportion varies with respect to $x$ at different $\eta$.

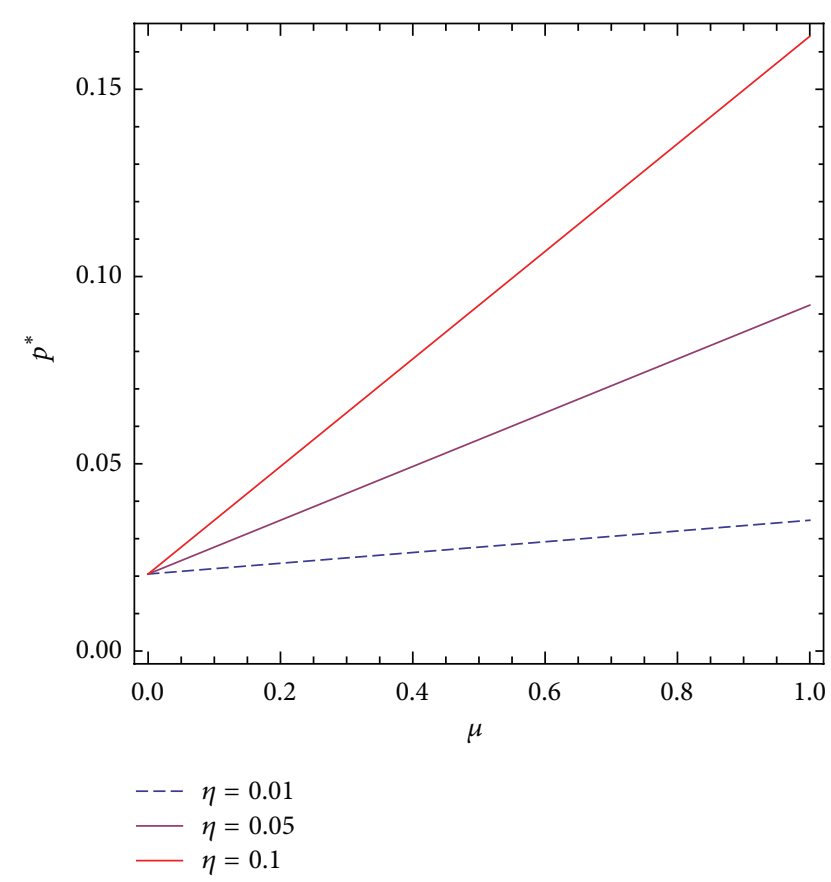

FIgURE 6: The optimal reinsurance proportion varies with respect to $\mu$ at different $\eta$.

asset will be higher. Under the condition of having positive yields higher than the interest rate, if the fluctuation of risk investment is more smaller, the share of risk investment will increase more rapidly. If risk asset fluctuates greatly, despite having higher investment income than the risk-free asset, the growth of investment proportion is still very small. 


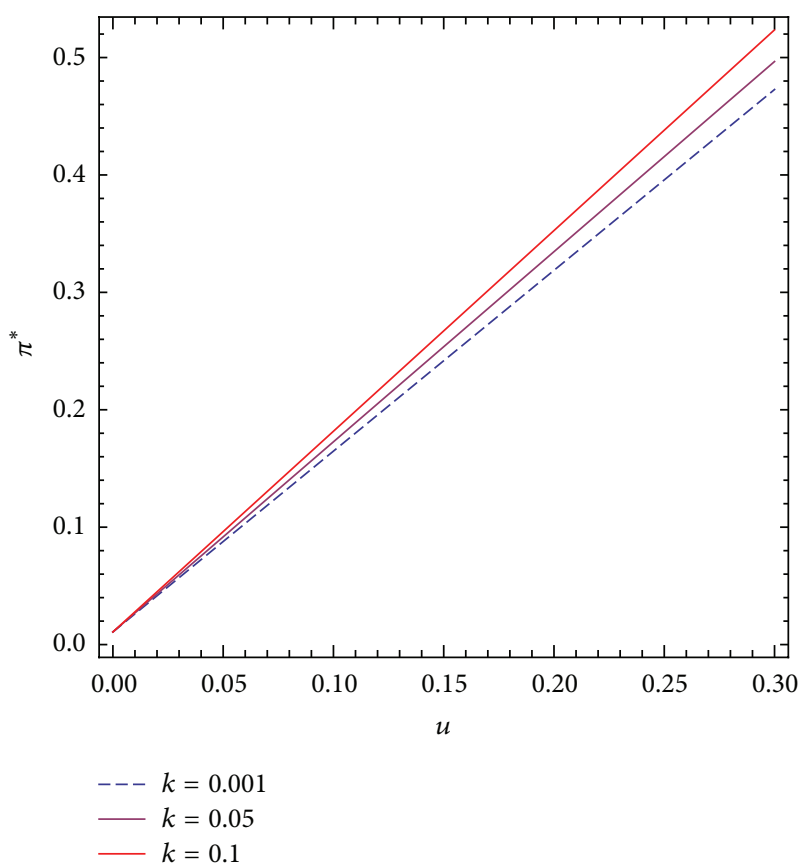

FIGURE 7: The optimal investment strategy varies with respect to $u$ at different $k$.

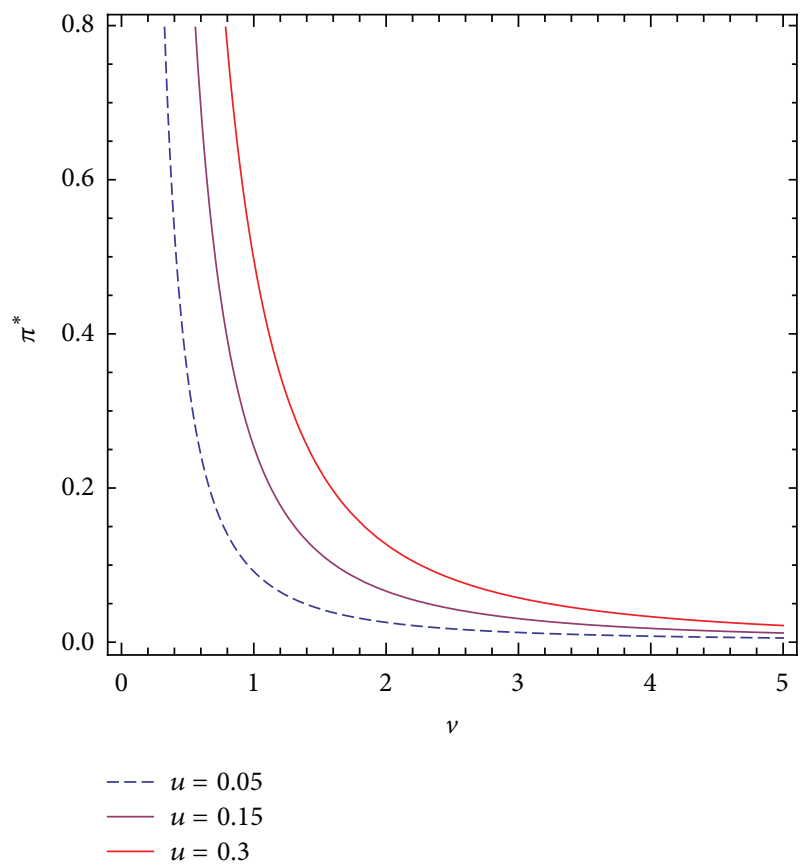

Figure 8: The optimal investment strategy varies with respect to $v$ at different $u$.

It is worth to say that the above analysis and graphics just focus on some key parameters. In fact, the influence of other parameters is also very important. The analysis based on different views can lead to different conclusions, and the work here is just serving as a modest spur to induce someone to come forward with his valuable contributions.

\section{Conclusion}

Based on the viewpoint of interaction between insurance company and policy-holder, we introduce reward budget into the insurance risk management process in this paper, which makes the insurer's income to be dynamic. Some results are obtained under the Vasicek stochastic interest rate model; the results of which also can be generalized to some other interest rate models.

\section{Conflict of Interests}

The author declares that there is no conflict of interests regarding the publication of this paper.

\section{Acknowledgments}

This research was supported by National Natural Science Foundation of China (Grant no. 11301376).

\section{References}

[1] Y. Cao and J. Xu, "Proportional and excess-of-loss reinsurance under investment gains," Applied Mathematics and Computation, vol. 217, no. 6, pp. 2546-2550, 2010.

[2] Y. Cao and X. Zeng, "Optimal proportional reinsurance and investment with minimum probability of ruin," Applied Mathematics and Computation, vol. 218, no. 9, pp. 5433-5438, 2012.

[3] M. Grasselli, "A stability result for the HARA class with stochastic interest rates," Insurance: Mathematics and Economics, vol. 33, no. 3, pp. 611-627, 2003.

[4] J. Li and R. Wu, "Optimal investment problem with stochastic interest rate and stochastic volatility: maximizing a power utility," Applied Stochastic Models in Business and Industry, vol. 25, no. 3, pp. 407-420, 2009.

[5] T. Pang, "Stochastic portfolio optimization with log utility", International Journal of Theoretical and Applied Finance, vol. 9, no. 6, pp. 869-887, 2006.

[6] C. Munk and A. Rubtsov, "Portfolio management with stochastic interest rates and inflation ambiguity," Annals of Finance, vol. 10, no. 3, pp. 419-455, 2014.

[7] R. Korn and H. Kraft, "A stochastic control approach to portfolio problems with stochastic interest rates," SIAM Journal on Control and Optimization, vol. 40, no. 4, pp. 1250-1269, 2002.

[8] D. Hainaut, "Dynamic asset allocation under VaR constraint with stochastic interest rates," Annals of Operations Research, vol. 172, no. 1, pp. 97-117, 2009.

[9] O. Vasicek, "An equilibrium characterization of the term structure," Journal of Financial Economics, vol. 5, no. 2, pp. 177-188, 1977.

[10] R. C. Merton, "Optimum consumption and portfolio rules in a continuous-time model," Journal of Economic Theory, vol. 3, no. 4, pp. 373-413, 1971.

[11] H. U. Fleming and H. M. Soner, Controlled Markov Processes and Viscosity Solutions, Springer, Berlin, Germany, 1993.

[12] G. Guan and Z. Liang, "Optimal management of DC pension plan in a stochastic interest rate and stochastic volatility framework," Insurance: Mathematics and Economics, vol. 57, pp. 5866, 2014. 
[13] G. Guan and Z. Liang, "Optimal reinsurance and investment strategies for insurer under interest rate and inflation risks," Insurance: Mathematics \& Economics, vol. 55, pp. 105-115, 2014.

[14] A. Gu, X. Guo, Z. Li, and Y. Zeng, "Optimal control of excessof-loss reinsurance and investment for insurers under a CEV model," Insurance: Mathematics and Economics, vol. 51, no. 3, pp. 674-684, 2012. 


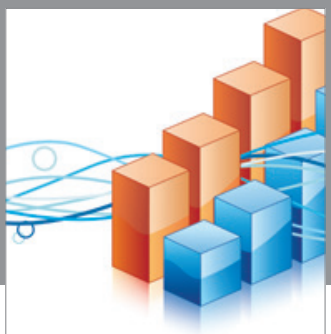

Advances in

Operations Research

vatem alat4

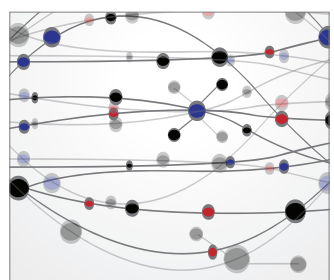

\section{The Scientific} World Journal
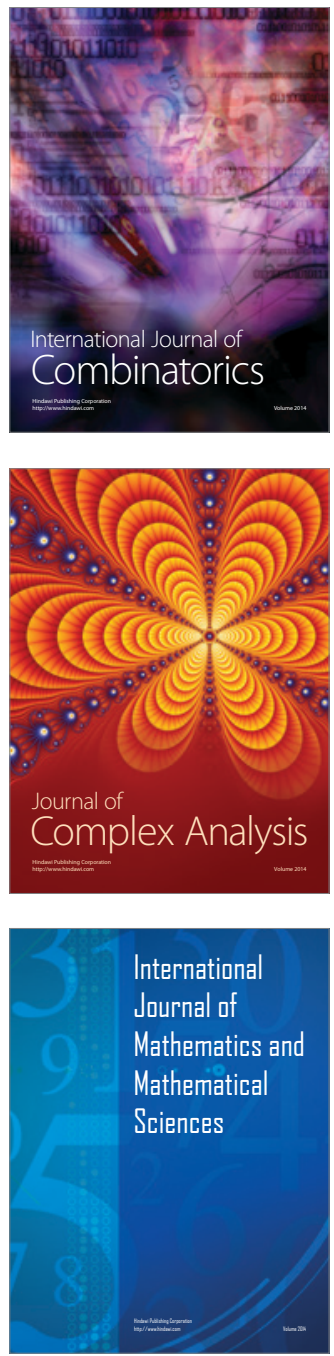
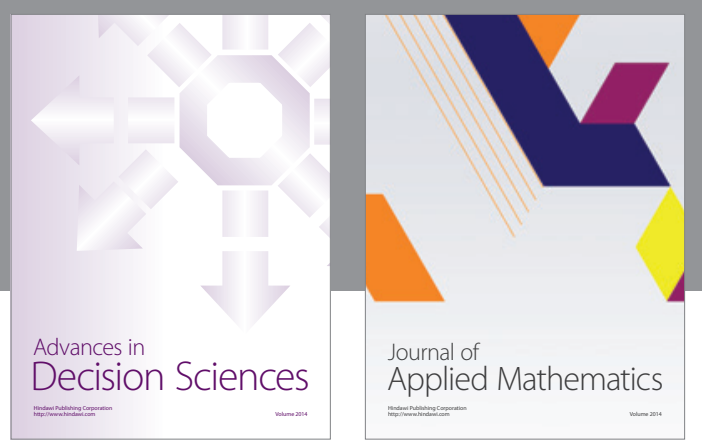

Algebra

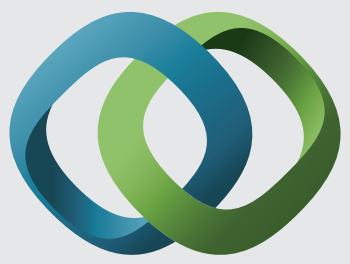

\section{Hindawi}

Submit your manuscripts at

http://www.hindawi.com
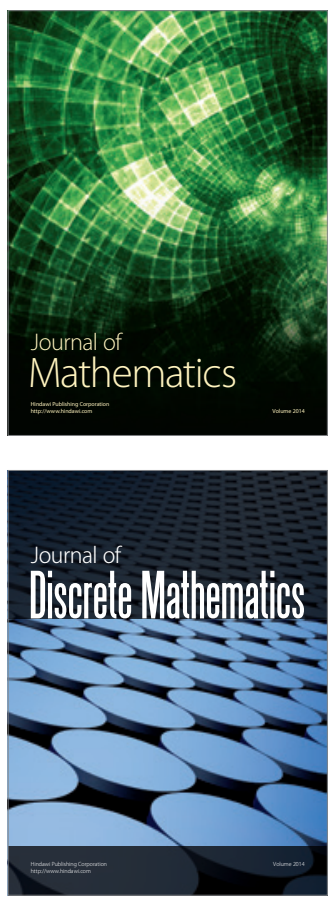

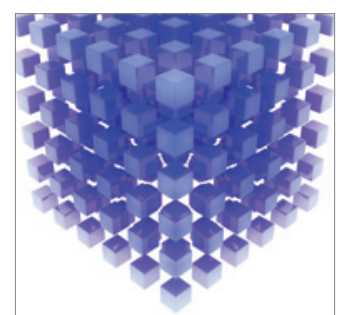

Mathematical Problems in Engineering
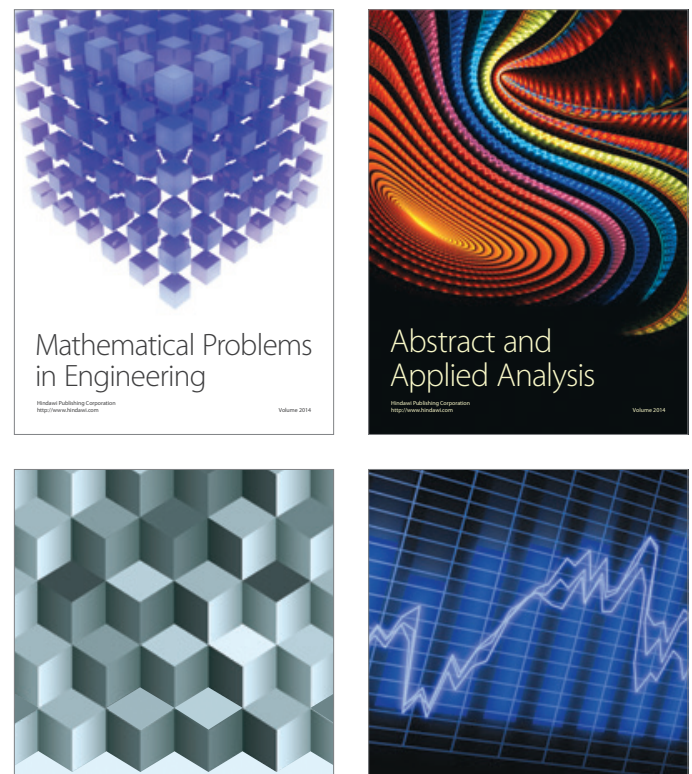

Journal of

Function Spaces

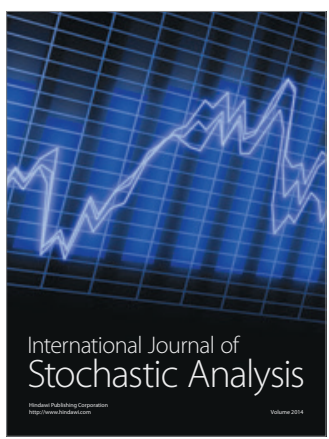

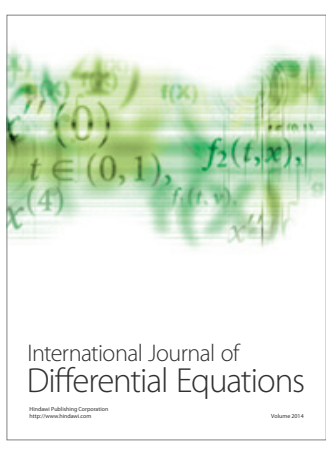
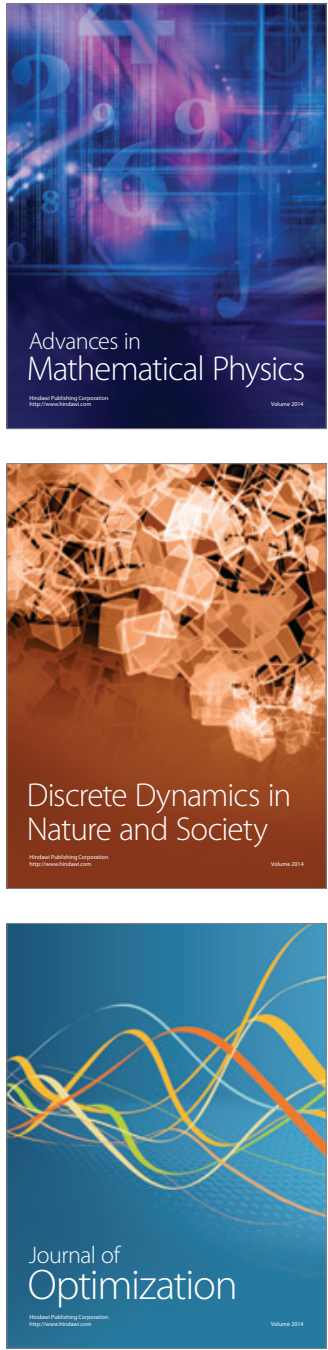